\title{
AN INTRODUCTION TO IBM GENERAL BUSINESS SIMULATION ENVIRONMENT
}

\author{
Wei Wang \\ Jin Dong \\ Hongwei Ding \\ Changrui Ren \\ Minmin Qiu \\ IBM China Research Lab \\ Zhongguancun Software Park \\ Beijing 100193, CHINA
}

\author{
Young M. Lee \\ Feng Cheng
}

IBM T. J. Watson Research Center

1101 Kitchawan Road

Yorktown Heights, New York 10598, USA

\begin{abstract}
IBM General Business Simulation Environment (GBSE) is a supply chain simulation tool developed by IBM China Research Lab. It can capture supply chain dynamics with finest level of granularity and provides great insights to a supply chain's real operations. GBSE is designed for tactical level decision making; it is proper for supply chain what-if analysis and risk analysis. GBSE implements multiple supply chain processes to considerable details, such as order handling process, inventory control process, manufacturing process, transportation process, procurement process, and planning. The environment is created as a desktop software tool based on Eclipse platform. The backbone framework consists of Presentation Layer, Controller Layer, Service Layer, and Data Layer.
\end{abstract}

\section{INTRODUCTION}

Simulation technology has been widely used as one of the most powerful technologies for analyzing and improving enterprises' supply chain management and logistics operations. Although analytical methods such as optimization play very key roles in the area of supply chain management and logistics, they usually need to make lots of assumptions to simplify the model, which limits their application in tactical level and operational level of decision making. On the opposite, with simulation technology, analyst can deal with more details and give more comprehensive results. Moreover, simulation tools usually have strong visualization capability and provide analysts an intuitive view of their supply chain.

IBM China Research Lab developed a supply chain simulation tool, named General Business Simulation Environment (GBSE), which is a flexible and powerful software tool to help supply chain practitioners to model, simulate and analyze their supply chains. GBSE is previously a part of IBM SmartSCOR (Dong 2006), and it is usually used for making tactic-level decisions, meanwhile it has interfaces linking with external tools for modeling work at strategic or operational level.

Simulation is basically an evaluation technology, and it must have pre-defined inputs to run an experiment. So it is very common to integrate it together with optimization. External optimization engines or tools can be leveraged by GBSE. For instance, logistics network optimization tool can be used to provide an optimal-configured network, which is used as the input for simulation. Another example is its capability to call external supply chain planning engine during the simulation to generate plan and place purchase orders.

From IT perspective, GBSE is a software tool developed by pure Java. It is based on Eclipse platform and implemented as an Eclipse RCP (Rich Client Platform).

Simulation also has its shortages. First, it is a challenge for analyst to determine the proper level of granularity. Secondly lots of skills are required to create a simulation model, and sometimes it is necessary to write programming codes for special scenarios. Thirdly, it is not easy to validate the model and convince clients that the model can reflect the real situations. Fourthly, lots of data are required to perform simulation; if not all data are available, the simulation model needs to be modified accordingly. Fifthly, it is very time-consuming to run a simulation; usually it takes several hours to run one medium-size scenario. Although GBSE can't resolve all these issues, it can help analysts to create simulation model easier than ever; the running speed is also tuned and optimized carefully.

In the remainder part of this paper, section 2 gives a literature review on existing supply chain simulation tools and libraries. Section 3 introduces the tool architecture. Section 4 gives some details on IT implementation of the tool. Section 5 discusses several aspects of the supply 


\section{Wang, Dong, Ding, Ren, Qiu, Lee, and Cheng}

chain simulation model. Conclusions and perspectives are given in section 6 .

\section{REVIEW OF SUPPLY CHAIN SIMULATION}

Simulation has been widely used in supply chain management and logistics area, and several surveys have been done in this area. Kleijnen (2003) provides a survey of simulation in supply chain management and reviews four types of simulation, namely spreadsheet simulation, system dynamics, discrete event simulation (DES), and business games. Terzi and Cavalieri (2004) provides a comprehensive review; local simulation and parallel and distributed simulation paradigms are also compared. Normally simulation methods fall into three categories: local simulation and distributed simulation. Distributed simulation has many advantages, and it can leverage computation resources from multiple machines to handle larger problems within shorter time. But so far, in supply chain simulation area, local simulation is still popular because of its simplicity. In local simulation, Monte-Carlo Simulation (MCS) and DES are two most important methods. MCS is generally a static method, and usually being performed in spreadsheet based tools. MCS is a light-weight method and very useful for creating some high-level models and getting preliminary results. DES is heavier and can handle more details. IBM GBSE is a tool based on DES.

A bunch of general-purpose simulation tools can be used to simulate supply chain, such as Arena, AnyLogic, AutoMod, etc. When using these tools, analysts usually create supply chain models with a set of pre-defined logic elements, like CreateNode, DecisionNode, DisposeNode, etc. On one hand, Analysts can create a model much faster than using programming language; on the other hand, the model can be complex enough to cover real situations. This is the advantage to use general-purpose simulation tools. Most of these tools have separate views for logic and presentation. Logic view shows the internal simulation logic with flow charts, decision trees, etc; while presentation view shows animations, charts, maps, etc. So analysts can edit internal logic in logic view and draw fancy diagrams in presentation view; this is very useful and powerful.

There are also some simulation tools designed specifically for supply chain. IBM Supply Chain Simulator is a software tool that can help a company or a group of companies make strategic business decisions about the design and operation of its supply chain (Bagchi 1998). EasySC is a simulation platform for understanding supply chains through studying the impact of stochastic demands, logistics decisions and production policies on key performance measures (Liu 2004). Supply Chain Guru (Supply Chain Guru 2008) is a supply chain simulation and optimization tool for enterprise strategic planning and targeted supply chain performance improvements. By using these tools, analysts can create models with supply chain language, like adding a warehouse node, connecting two distribution centers with routes, etc. GBSE itself is also this kind of supply chain simulation tool. Comparing with existing tools, GBSE is highly extensible and flexible, and it is stable to simulate large-scale supply chains. GBSE also has friendly user interfaces, which make supply chain modeling and simulation much easier.

Except the tools, some frameworks are also developed for supply chain simulation. Swaminathan (1998) describes a supply chain modeling framework, in which supply chain models are composed from software components that represent types of supply chain agents, their constituent control elements, and their interaction protocols. Rossetti and Chan (2003) discusses the design, development and testing of a prototype object-oriented framework for performing supply chain simulation. Rossetti (2006) discusses a JSL (Java Simulation Library) based object-oriented framework for simulating multi-echelon inventory systems.

A bunch of simulation libraries are also available, they can be leveraged when building simulation tools to avoid inventing wheels. Some famous and powerful libraries, such as Repast (Repast 2008) and Scalable Simulation Framework (SSF 2008), are available from internet.

\section{ARCHITECTURE}

Since GBSE is targeted as a generic purpose business simulation environment, the architecture is carefully designed and results in a very flexible framework and platform. To make the it more clear, four layers are introduced as figure 1. From bottom to top, respectively: data layer, controller layer, service layer, and presentation layer. Data Layer manages all the data in simulation; Service Layer defines all the simulation processes; Controller Layer implements the simulation engine; Presentation Layer is the interface to simulation analyst.

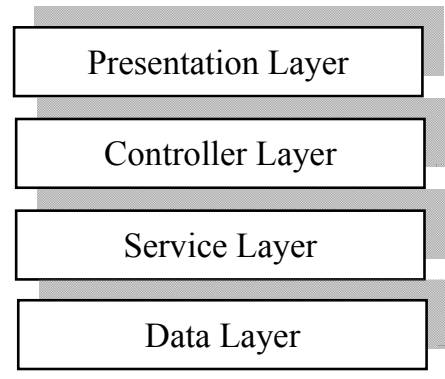

Figure 1: GBSE Architecture

\subsection{Data Layer}

Data layer manages all the simulation data, including both configuration data, runtime data and report data. A nontrivial simulation study always involves huge scale of data, for instance, customer orders, daily transactions, ocean and 


\section{Wang, Dong, Ding, Ren, Qiu, Lee, and Cheng}

truck shipments, etc. It is usually very time-consuming to access and handle these data, this causes it quite slow to run a simulation. So an efficient data access solution is important to the success of simulation study.

In GBSE, the data access layer is built upon a database system. Per our experiences, using simple model files to hold simulation models often creates scalability issues particularly since we always need to handle industry-scale data. It is very easy to get out of memory and it is also quit slow to access data in simple model file.

Data import and export are supported. Currently Microsoft Excel files and CSV (Comma Separated Values) files are supported.

\subsection{Service Layer}

Service layer contains simulation services. A simulation service is the smallest unit in a simulation run.

Four types of data are associated with a service: configuration data, input data, output data, and state data. Each service has its internal transactions to operate on inputs and generate outputs. The behavior of services are controlled by their configuration data, and the runtime status is stored in state data.

Data layer is leveraged by service layer to manage the four types of data. Simulation logics are implemented as one service or combination of several services. Services can be grouped as service bundles.

\subsection{Controller Layer}

Controller layer is responsible for scheduling service events and dispatching messages between services. A discrete event simulation (DES) engine and a simulation bus are implemented in this layer.

The DES engine is the heart of the controller layer. An event list is maintained in the engine, and events are scheduled to happen at certain time point as services request. In a non-trivial simulation study, millions of events can be in place at the same time, so it could be a big challenge for the DES engine to handle them effectively. GBSE has a built-in high-performance DES engine. Meanwhile the interface is open and it is also possible to leverage another external DES engine.

The simulation bus is the backbone of the controller layer. All services are connected to the service bus and exchange messages through it.

\subsection{Presentation Layer}

Presentation layer is the interface to the end users. It helps users to build model, run simulation, and do analysis with reports.

There are two perspectives available. Modeling Perspective is used to create simulation model. Both diagram editor and table editor are provided. Running Perspective is used in simulation running process. Animation and moving charts are present to make the simulation more intuitive. And more important, it is extremely useful to validate the simulation process.

Functions in presentation layer include: (1) Create and edit simulation model; (2) import and export simulation data; (3) control simulation running; (4) monitor simulation running status; (5) generate and check simulation report.

\subsection{Templates}

So far we have introduced all the four layers in GBSE. To leverage them and create simulation models, we need to define templates. Template is a key concept in GBSE. A template is a module which consists of (1) simulation elements and (2) customized user interfaces; each simulation element consists of data definitions and services; services can be reused in several simulation elements.

As an example, in GBSE we can have a template for distribution network, and another template for banking system. In the distribution network template, simulation elements are distribution centers, warehouses, hubs, retails, routes, etc. And in banking system template, simulation elements are banking branches, ATMs, customers, etc. GBSE provides different palettes for different templates. After applying a template to a model, analysts can drag elements from the palette and drop them to the model. A uniform table editor is provided for editing data associated with each element.

In most cases, the default user interfaces provided by GBSE are good enough; while for some special cases, analysts can also create their own user interfaces in the template for editing data, displaying moving curve, etc.

\section{GBSE TOOL IMPLEMENTATION}

GBSE is a pure-Java application built upon Eclipse platform and implemented as an Eclipse RCP. Eclipse is an open platform comprised of extensible frameworks, tools and runtime for building, deploying and managing software across the lifecycle (Eclipse 2008).

To make it easier to use GBSE, we provides several different perspectives in GBSE. Perspective is a term in Eclipse platform, it stands for a kind of layout of the whole user interface. As two examples, figure 2 shows the modeling perspective, which is convenient for analysts to model the supply chain and fill in data; and figure 3 shows the running perspective, which is useful for launching the simulation and monitor the process through animation and moving curves.

A database should be in place to run GBSE. The choice of database is very flexible, any JDBC compatible database can be used by GBSE. For large scale simulation, 
we recommend IBM DB2 to achieve best performance. For small cases with demo purpose only, Apache derby is a good choice.

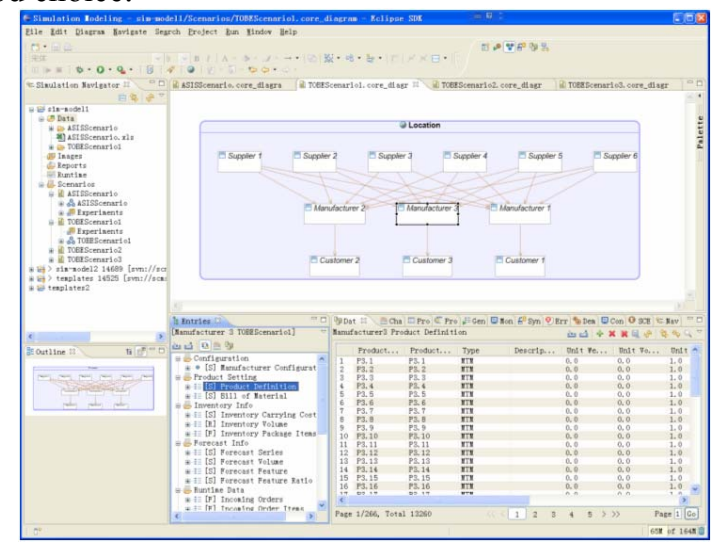

Figure 2 Modeling Perspective

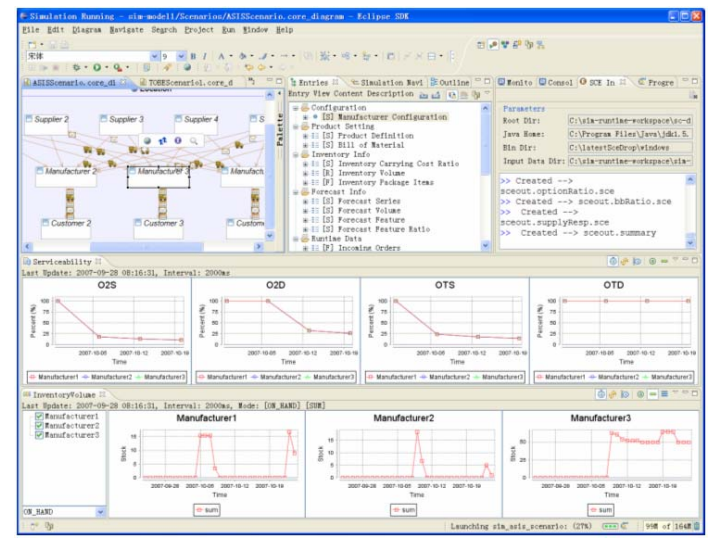

Figure 3 Running Perspective

\section{SUPPLY CHAIN SIMULATION MODEL}

The functionality of GBSE can be extended by all kinds of GBSE templates. Supply Chain Simulation Template is the most important one. It implements supply chain model at tactical level, and allows analysts to create a multi-period and multi-echelon supply chain model. This model is designed for generic-purpose and will be applicable for most supply chain scenarios. For some extreme cases, more flexibilities are provided to experienced analysts, who can enhance the model through several extension points of the model. Details of this simulation model is introduced in the next several sections.

\subsection{Data Structure}

This section introduces data structure in the model. All these data are managed in data layer and stored in database. Collecting and cleaning up data is the one of the most time-consuming part of simulation modeling, and the success of a simulation study depends on the quality of data directly.

\subsubsection{Calendar Data}

GBSE adopts a multi-period simulation model. A period can be modeled as a day, a week, a month, a quarter, or even a year. The choice of period definition does depend on the expected model granularity. Smaller period length means finer granularity and of course more requirements on data.

The simulation configuration data can be different for different periods. This is useful to model time-changing parameters like seasonality demand. The simulation outputs of different periods can be collected separately so as to show the trends to the analysts.

Calendar data are also critical for building supply chain plan. Different planning horizon can lead to different planning results.

\subsubsection{Product Data}

Product represents finished goods, raw materials, intermediate products, etc. In a simulation study, usually it is not realistic to model all the products in the supply chain, so analysts need to select a proper subset carefully since most configuration data are specific to certain kind of products. General information for products should be provided, such as weight, dimension, brand, etc.

If manufacturing processes are taken into account, analysts also need to determine bill of material (BOM), which defines the composition relationships between different levels of products. Again, for a specified products, usually not all parts can be considered. Analysts should select parts based on certain rules, such as selecting most expensive or heaviest parts.

\subsubsection{Network Data}

Supply chain network consists of nodes and links. In GBSE simulation model, we define 3 kinds of nodes (Customer, Supplier, and Facility) and 1 kind of link (Lane).

Supplier nodes are the source of supply, and they are the origins of a supply chain. Since there are no upstream nodes for suppliers, their inventories are replenished from the air. But supply capacity is still taken into account, this limits the supply volume within specified periods.

Customer nodes are the source of demand, and they are final destinations of a supply chain. In a real supply chain, there could be up to millions of individual customers; of course there is no need to model them one by one, and proper aggregation must be conducted here. Depending on the size of the supply chain, customers can be modeled as big as a country, or as small as a shop.

Facility nodes are internal sites which are taken into account with details in a simulation study. Warehouses, distribution centers, factories, retailers, wholesalers are all modeled as facility nodes. Facilities are modeled with 
more details than suppliers and customers, and more attentions need be paid on them.

Lanes are physical directed connections between nodes. Inbound lands connect suppliers and facilities; outbound lanes connect facilities and customers; inter-facility lanes connect facilities and facilities. There should be no more than one lane between two nodes, but there can be multiple transportation modes associated with one lane.

If geographical information is provided, such as latitude and longitude, the supply chain network can be displayed in a geographic information system (GIS) embedded in GBSE. This gives analysts an intuitive view of the entire network.

\subsubsection{Configuration Data}

After calendar, product and network are modeled, it is time to collect data for each node and link in the network. Analysts need to collect demand data, cost data, capacity data, lead time data, policy data, etc.

Demand data are defined for Customer Nodes. Usually only aggregated demand are available. GBSE can split the aggregated demand into a number of orders.

Cost data include one-time transition cost, fixed cost and variable cost. One-time transition cost is only taken into account when some existing facilities are closed or some potential facilities are opened. Fixed cost is not associated with certain kind of product, while variable cost depends on product volume. In a multiple periods model, both fixed cost and variable cost are associated with periods, which means for different periods, the cost can be different.

Capacity data consists of supply capacity, manufacturing capacity, storage capacity, handling capacity, transportation capacity.

Lead time data are applied to supply chain processes with long processing times. Such as manufacturing lead time, transportation lead time, handling lead time, etc. Usually lead time is defined as a random number. A powerful expression builder (Figure 4) is proved in GBSE for analysts to input them easily.

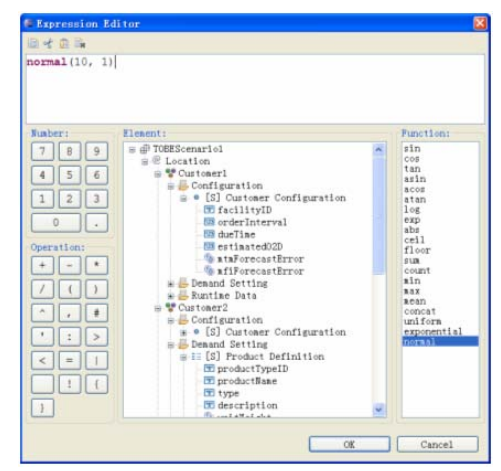

Figure 4 Expression Builder
Policy data are parameters to different policies. Like batch size for transportation policy.

Some other data such as customer order interval, shipping shrinkage, manufacturing yield, budget, time threshold can also be defined. If there are no such requirements in simulation study, it is convenient for analysts to keep them as default values.

\subsection{Supply Chain Processes}

This section introduces supply chain processes taken into account. A process is a working unit at operational level; it has its inputs and outputs, as well as internal logic to handle inputs and generate outputs. Meanwhile, processes can be controlled by local parameters and global parameters. In GBSE, All these processes are implemented as services in service layer.

\subsubsection{Resource Management}

Resources management plays a key role to the entire simulation model. In supply chain context, resources can be workers, trucks, drivers, handling equipments, etc. They have limited capacity but are necessary to perform some kind of tasks. GBSE implements a generic resource management framework and it is used in several aspects of supply chain processes. The key components are resource and resource pool. A task can request a resource from the pool, and return the resource to the pool when it is finished. Some tasks can also consume some resources and the pool is in charge of resource replenishment.

\subsubsection{Customer Order Generating Process}

Customer Order Generating Process is only applicable for Customer Node. Usually aggregated demand data are available for those customers. So in simulation, the demand will be split into several batches; in each batch, one or more customer orders are generated and sent to corresponding facilities.

Forecast errors are applied to order quantity to demonstrate demand uncertainty. Since the demand is split into a few batches, the forecast error can be applied to either the total demand of the period, or the order quantity in one batch. These two methods can have very big differences.

\subsubsection{Order Processing}

When an order enters a facility, first it will be preprocessed, which is usually finished in a short time, and then the order is put into an prioritized order queue. The order queue will be checked periodically. For each round of check, some orders will be released and leave the order queue to get processed. Orders with higher priority will leave the queue earlier than orders with lower priority. 


\section{Wang, Dong, Ding, Ren, Qiu, Lee, and Cheng}

Availability check is performed for each order to determine whether it can be released. This process is very complex, and the processing logic varies in different supply chain. Usually on-hand stock is checked first. If it is not enough to fulfill the order, in-transit stock will be checked; in this step, the due time needs to be estimated and compared with the arrival time of in-transit shipments. If it still not available, some special methods will be tried, like placing urgent purchase orders. If all the trials fail, the order will stay in the order queue and waiting for the next round of check.

For Supplier Node in GBSE, the inventory is unlimited, so the order processing process becomes much simpler. While supply capacity still needs to be taken into account, which limits the number of released orders during a time period.

\subsubsection{Inventory Process}

Inventory plays a key role in GBSE, and it can be regarded as the control center. As a matter of fact, products fall into two categories, in-source and out-source. For in-source products, the replenishment is built from the manufacturing process in the same facility; for out-source products, the replenishment is from external suppliers via procurement process.

Inventory control is applied to each product in each facility. User can specify periodical review or continuous review. Several typical inventory control policies are implemented including $(\mathrm{R}, \mathrm{Q})$ and $(\mathrm{s}, \mathrm{S})$. These parameters can be set for each product and each period.

If required, handling process can be modeled, which represents the tasks to move products into the storage area or move them out. This will involve resource framework to manage the handling equipments.

\subsubsection{Procurement Process}

Procurement process is in charge of purchasing products from upstream facilities or suppliers. Procurement policies fall into two categories: single sourcing and multiple sourcing. Single sourcing policy is pretty straightforward, analyst needs to define supplier for each product in each period. For multiple sourcing policy, things get more complex. A set of methods are defined in GBSE for supplier selection. As an example, analysts can define the volume proportion for each supplier and each product.

Procurement process can be triggered by procurement plan and inventory signals. Procurement plan defines the time and quantity of each procurement task, which allows analysts the complete control of procurement process. In contrast with that, inventory control process can also trigger procurement tasks for out-source products when the inventory level is lower than a predefined reorder point.
Procurement tasks can be consolidated. Purchase orders to the same supplier will be grouped based on the desired delivery date.

\subsubsection{Manufacturing Process}

GBSE is focused on discrete-manufacturing area, and an assembling process is implemented to model the consuming of raw materials and creating intermediate products and finished goods.

Similar to procurement process, manufacturing process can also be triggered by manufacturing plan and inventory signals. Manufacturing plan consists of a set of manufacturing tasks; each task has its time and quantity. Inventory signal is sent from inventory process for insource products, which has been discussed in the inventory section.

Manufacturing capacities are defined to restrict the maximum manufacturing volume for specified products and periods. On the other hand, analysts also can define manufacturing resources, this stands for machines, workers, etc. When corresponding resources are not available, manufacturing process will be pending.

An overall manufacturing cost is calculated in this process. This includes direct labor cost, in-direct labor cost, energy consumption, and all other variable costs during manufacturing.

\subsubsection{Transportation Process}

Transportation process happens on inbound lanes, outbound lanes, and inter-facility lanes.

Several types of transportation modes are supported in GBSE, like LTL (Less-than-Truckload), TL (Truckload), ocean shipping, train, and air shipping, analysts can also define their own modes. Transportation time, cost, as well as the desired service level, should be set for each mode.

Transportation tasks can be consolidated, which can save a lot of transportation costs. These tasks are organized and grouped based on customers and due time, and then they are sent to customers in batches.

\subsubsection{Planning Process}

To enable BTP (Build-to-Plan) process, it is necessary to run MRP process during the simulation. Several parameters and system status will be taken into account by MRP process. From demand point of view, average customer demands in the next several periods should be provided; from inventory point of view, backlog, in-transit inventory, and on-hand inventory should be calculated together; from time point of view, supply lead time and delivery lead time should be estimated. With all the three kinds of data as input, procurement quantity and procurement time can be determined. 
A planning engine is developed in GBSE based on MRP explosion, with the interfaces clearly defined to allow the option of using external engines.

\subsection{Service Level}

One important target of GBSE is to balance service level and total cost. Service Level is measured from time aspect and percentage aspect.

Time related metrics show supply chain responsiveness. Analysts would like to see order cycle time, average assembling time, average transportation time, etc. And it is also useful to check the average waiting time for an order queue or a resource pool.

Percentage related metrics represent supply chain effectiveness. Analysts would like to see the order fill rates, procurement fulfill rates, out-of-stock rates, etc.

\section{CONCLUSION}

This paper introduces IBM GBSE, a powerful integrated supply chain simulation environment developed by IBM China Research Lab. It has been applied for both IBM internal and external supply chains to evaluate several key performance metrics and optimize the operations. The tool is designed for tactical level of decision making, and it supports wide range of supply chain processes from high level planning process to detailed order handling process. Simulation analysts can use it to conduct several kinds of what-if analysis and risk analysis in supply chain context, so as to make correct decisions more effectively.

\section{REFERENCES}

Bagchi, S., S. J. Buckley, M. Ettl, and G. Lin. 1998. Experience using the IBM Supply Chain Simulator. In Proceedings of the 1998 Winter Simulation Conference.

Eclipse platform 2008. Available via $<\mathrm{http}: / /$ www.eclipse.org/>

Dong, J., H. Ding, C. Ren, and W. Wang. 2006. IBM SmartSCOR - A Scor Based Supply Chain Transformation Platform. In Proceedings of the 2006 Winter Simulation Conference.

Kleijnen, J.P.C. 2003. Supply Chain Simulation: A Survey. International Journal of Simulation and Process Modeling 103:1-20.

Liu, J., W. Wang, Y. Chai, and Y. Liu. 2004. Easy-SC: A Supply Chain Simulation Tool. In Proceedings of the 2004 Winter Simulation Conference.

Repast 2008. Available via $<$ http://repast.sourceforge.net/>

Rossetti, M. D. and H. T. Chan. 2003. A Prototype ObjectOriented Supply Chain Simulation Framework. In Proceedings of the 2003 Winter Simulation Conference.
Rossetti, M. D., M. Miman, V. Varghese, and Y. Xiang. 2006. An Object-Oriented Framework for Simulating Multi-echelon Inventory Systems. In Proceedings of the 2006 Winter Simulation Conference.

Scalable Simulation Framework (SSF) 2008. Available via $<$ http://www.ssfnet.org/homePage.html $>$

Supply Chain Guru 2008. Available via $<\mathrm{http}: / /$ www.llamasoft.com/>

Swaminathan, J. M., S. F. Smith, and N. M. Sadeh. 1998. Modeling Supply Chain Dynamics: A Mutliagent Approach, Decision Sciences 29:607-632.

Terzi, S., and S. Cavalieri. 2004. Simulation in the Supply Chain Context: A Survey. Computers in Industry 53:316.

\section{AUTHOR BIOGRAPHIES}

WEI WANG is a Researcher at IBM China Research Laboratory. He joined IBM Research in 2005 after receiving his master degree in Control Science and Engineering from Tsinghua University in Beijing, P. R. China. Currently, his research interests include supply chain simulation and optimization, performance management, and business process management. His e-mail address is <wangwcrlecn.ibm. com>.

JIN DONG, Manager of Supply Chain Management and Logistics Research in IBM China Research Laboratory. He received his Ph.D. degree in Tsinghua University from P.R. China in 2001. Before joined IBM, he was the Research Assistant Professor in Industrial Engineering Department of Arizona State University in USA. His e-mail address is <dongjin@en. ibm. com>.

HONGWEI DING is a Researcher at IBM China Research Laboratory. He received his Ph.D. in Automation from INRIA (French National Institute of Computer Science \& Control), France. Before joined IBM, he was a researcher at INRIA. His research interests include supply chain modeling, optimization and simulation. His e-mail address is $<$ dinghwe cn. ibm. com>.

CHANGRUI REN is a Researcher at IBM China Research Laboratory. He joined IBM Research in 2005 after receiving his Ph.D. degree in Control Science and Engineering from Tsinghua University in Beijing, P. R. China. His research interests include supply chain management, logistics network design, performance management, and business process management. He is currently working on an end-to-end supply chain transformation methodology and tool. His e-mail address is $\langle$ rencrecn. ibm. com >.

MINMIN QIU is a Researcher at IBM China Research Laboratory. He joined IBM Research in 2006 after receiving his Ph.D. degree in Industrial Engineering from Tsing- 
hua University in Beijing, P.R. China. His research interests include inventory optimization, machine learning, supply chain optimization and simulation. His e-mail address is $<q i u m m @ e n$. ibm. com>.

YOUNG LEE is a Research Staff Member in the mathematical science department of IBM's T.J. Watson Research Center. Dr. Lee received B.S., M.S., and Ph.D. degrees in chemical engineering from Columbia University. He joined the IBM Research Division in 2002, and has been working in the areas of supply chain simulation and optimization. Prior to joining IBM, he had worked for BASF for 14 years, where he had founded and managed the Mathematical Modeling Group, and led development of numerous optimization and simulation models for various logistics and manufacturing processes. His research interest includes simulation and optimization of supply chain, manufacturing, services and business processes. His email address is <ymleedus. ibm.com>.

FENG CHENG is a member of the Business Analytics and Mathematical Sciences Department at the IBM Thomas J. Watson Research Center, in Yorktown Heights, NY. He joined IBM Research Division in 1996 after receiving a Ph.D. degree in Operations Research from the University of Toronto. Dr. Cheng has worked on a wide range of research and business consulting projects in various areas including business process modeling, supply chain simulation and optimization, and operational risk management. He has published research papers in the top-ranked academic journals of the related fields. His e-mail address is $<$ fcheng@us.ibm.com>. 